\title{
SÍNTESIS DE LÍPIDOS DE LA MICROALGA Nannochloropsis oculata PARA SU USO POTENCIAL EN LA PRODUCCIÓN DE BIODIÉSEL
}

\author{
María del Rosario MARTÍNEZ MACIAS*, Reyna Guadalupe SÁNCHEZ DUARTE, \\ Edna Rosalba MEZA ESCALANTE, Ruth Gabriela ULLOA MERCADO y Jorge SALDÍVAR CABRALES
}

Instituto Tecnológico de Sonora. Calle 5 de febrero 818 sur, Colonia Centro, Ciudad Obregón, Sonora, México, C. P. 85000

*Autor para correspondencia: maria.martinez@itson.edu.mx

(Recibido junio 2015; aceptado agosto 2016)

Palabras clave: ácidos grasos, productividad, luz, biocombustibles

\section{RESUMEN}

Las microalgas por su alto contenido de lípidos, son consideradas como fuente potencial en la obtención de biodiésel. En este estudio se evaluó la productividad de biomasa, lípidos y perfil de ácidos grasos en el cultivo de la microalga Nannochloropsis oculata bajo diferentes intensidades de luz. La microalga $N$. oculata fue cultivada a $25^{\circ} \mathrm{C}, \mathrm{pH}$ de 8 e intensidades de luz de $80,200,400$ y $650 \mu \mathrm{mol} \mathrm{E} / \mathrm{m}^{2} / \mathrm{s}$, en medio $\mathrm{f} / 2$ de Guillard, adicionado cada $72 \mathrm{~h}$. Los resultados indicaron que la intensidad de luz favoreció la densidad celular y la productividad de la biomasa, obteniéndose hasta $175 \pm 1.83$ y $148 \pm$ $1.97 \times 10^{6} \mathrm{cel} / \mathrm{mL}$ y tasas de crecimiento de $0.3432 \pm 0.08$ y $0.3299 \pm 0.021 / \mathrm{d}$ a irradiancias de 650 y $400 \mathrm{~mol} \mathrm{E} / \mathrm{m}^{2} / \mathrm{s}$, respectivamente. La acumulación de lípidos presentó el mismo comportamiento, a $650 \mu \mathrm{mol} \mathrm{E} / \mathrm{m}^{2} / \mathrm{s}$ alcanzó un valor de $44.44 \pm 0.76 \%$, el doble de lo obtenido a $80 \mu \mathrm{mol} \mathrm{E} / \mathrm{m}^{2} / \mathrm{s}$. Se logró una productividad de $0.040 \pm 0.0005 \mathrm{~g} / \mathrm{L} / \mathrm{d}$ a $650 \mu \mathrm{mol} \mathrm{E} / \mathrm{m}^{2} / \mathrm{s}$, siendo ocho veces mayor que la obtenida a $80 \mu \mathrm{mol} \mathrm{E} / \mathrm{m}^{2} / \mathrm{s}$. Esto puede deberse a que a altas irradiancias se favorece la fotosíntesis, con lo que se incrementá la acumulación de lípidos. No se encontraron diferencias significativas en contenido de ácidos grasos saturados e insaturados, sólo una reducción de $2.26 \%$ en el contenido del ácido eicosapentaenoico para el cultivo a $650 \mu \mathrm{mol} \mathrm{E} / \mathrm{m}^{2} / \mathrm{s}$. Con lo anterior, se incrementó el contenido de ácidos grasos saturados lo que permitió una mayor calidad en el biodiésel.

Key words: fatty acids, productivity, light, biofuels

\begin{abstract}
Microalgae are considered as a potential source for biodiesel due to its easy culture and lipid content. In this work, the productivity of lipids and fatty acid profiles of the microalgae Nannochloropsis oculata grown under different light intensities were evaluated. The microalga $N$. oculata was cultured at $25^{\circ} \mathrm{C}, \mathrm{pH} 8$ and light intensities of 80 , 200,400 and $650 \mu \mathrm{mol} \mathrm{E} / \mathrm{m}^{2} / \mathrm{s}$. Medium of Guillard $\mathrm{f} / 2$ was used to feed them every $72 \mathrm{~h}$. The results indicated that increasing the light intensity promotes cell density and biomass productivity. For the light intensities of 650 and $400 \mathrm{~mol} \mathrm{E} / \mathrm{m}^{2} \mathrm{~s}$, a cell density of $175 \pm 1.83$ and $148 \pm 1.97 \times 10^{6} \mathrm{cell} / \mathrm{mL}$ and a growth rate of $0.3432 \pm 0.08$ and $0.3299 \pm 0.021 / \mathrm{d}$, were obtained respectively. Lipid accumulation at a light intensity of
\end{abstract}


$650 \mu \mathrm{mol} \mathrm{E} / \mathrm{m}^{2} / \mathrm{s}$ was twice higher than the one obtained in culture at $80 \mu \mathrm{mol} \mathrm{E} / \mathrm{m}^{2} / \mathrm{s}$, reaching $44.44 \pm 0.76 \%$. Productivities showed the same behavior, it was increased at high light intensities, achieving $0.040 \pm 0.0005 \mathrm{~g} / \mathrm{L} / \mathrm{d}$, being eight times higher than the one obtained at a low light intensity. This behavior may be due to a high light irradiance, on which photosynthesis is increased, promoting lipid accumulation. No significant differences were found on the content of saturated and unsaturated fatty acids. Only a reduction of $2.26 \%$ was observed in the eicosapentaenoic acid content for culture at $650 \mu \mathrm{mol} \mathrm{E} / \mathrm{m}^{2} / \mathrm{s}$, thereby increasing the content of saturated and monounsaturated fatty acids, which allows a higher quality biodiesel.

\section{INTRODUCCIÓN}

En la actualidad el desmedido uso de hidrocarburos fósiles ha acentuado grandes descargas de sustancias tóxicas a la atmósfera, principalmente gases como el $\mathrm{CO}_{2}$. Lo que produce un efecto invernadero que contribuye con el calentamiento global y que impacta de manera nociva sobre la calidad del aire y la salud humana (Hu et al. 2008). La alta contaminación en el aire, el daño en la capa de ozono y la escasez de hidrocarburos, hacen que sea indispensable implementar nuevas tecnologías de generación de energías, capaces de abastecer las necesidades actuales de las poblaciones humanas. Una de las opciones es desarrollar técnicas de producción de biocombustibles, con lo que se evitaría el uso de suelo y se protegería además, la seguridad alimentaria del planeta.

El biodiésel se obtiene de cultivos oleaginosos (aceite vegetal o grasa animal), este biocombustible es candidato para reducir el consumo de combustibles fósiles. Sin embargo, no puede satisfacer ni una pequeña fracción de la demanda que existe (Chisti 2007). Las microalgas se consideran como la única materia prima prometedora en la producción de biodiésel, por su gran capacidad fotosintética (Wijffels y Barbosa 2010). Las microalgas pueden sobrevivir a altas concentraciones de $\mathrm{CO}_{2}$ y $\mathrm{NO}_{2}$, son responsables de la producción del $50 \%$ del oxígeno y del $50 \%$ de la fijación de carbono en el planeta (Garibay et al. 2009). Dichos organismos poseen tasas de conversión fotosintética más eficientes que las plantas y altos niveles de lípidos intracelulares que pueden utilizarse en la producción de biodiésel (Hossain et al. 2008). Entre las microalgas capaces de producir altas concentraciones de lípidos se encuentran Nannochloropsis oculata con contenidos que varían de 31 a 68 \% (Sánchez et al. 2008), Scenedesmus obliquus entre 10 y $17 \%$, incluso hasta $43 \%$ (Mandal y Mallick 2009), Dunaliella tertiolecta con cerca de $37 \%$ y Chlorella vulgaris con un 28 y $32 \%$ (Chacón et al. 2004).
La microalga Nannochloropsis oculata es reconocida por su valor nutricional como una excelente fuente de proteínas, carbohidratos, lípidos y vitaminas. Esta especie contiene cantidades elevadas de ácidos grasos poliinsaturados, especialmente ácido eicosapentaenoico (AEP), ácido araquidónico y ácido docosahexaenoico que son de gran relevancia en la nutrición de animales marinos, especialmente en el crecimiento y desarrollo de larvas de peces, moluscos y crustáceos (Sánchez et al. 2008). Adicionalmente, por su alto contenido de ácido palmítíco (C16:0) y palmitoleico (C16:1n-7), es una muy buena fuente para producir biodiésel.

Las microalgas mantienen la síntesis de lípidos, generalmente cuando se presenta deficiencia de nutrientes en el medio de cultivo o bajo condiciones de estrés ocasionadas por la reducción de otros constituyentes celulares (Hu et al. 2008). Las condiciones principales que influyen en la productividad de biomasa y lípidos son la temperatura (Sukenik et al. 2009), la salinidad (Pal et al. 2011, Su et al. 2011), la disponibilidad de nutrientes (Rezanka et al. 2011, Yeesang y Cheirsilp 2011, Yuan et al. 2011) y el color e intensidad de la luz (Simionato et al. 2011).

Se tienen antecedentes en Nannochloropsis sp., que el grado de insaturación de sus ácidos grasos sufre modificaciones con variaciones en la irradiancia, especialmente el porcentaje total de ácidos grasos n-3 (Arts et al. 2009). Estos ácidos son susceptibles a la oxidación durante su almacenamiento, por lo que su reducción potencializa la producción de biodiésel de alta calidad.

Por ser organismos fotosintéticos, las variaciones en la intensidad de luz en el cultivo de microalgas, afectan directamente a la productividad, se favorece la fotosíntesis a altas intensidades de luz y los nutrientes en cantidades suficientes permiten el desarrollo de biomasa. Aunque existe información acerca de la optimización de cultivos buscando la mejora en la productividad, tanto a nivel de formulación de medios de cultivo, parámetros de operación y diseño 
de biorreactores, estos factores son específicos para cada especie de microalga, con lo cual las posibilidades de llegar a un punto óptimo único para todas es poco probable. Cada especie de microalga tiene requerimientos específicos y se deben ir deduciendo conforme las necesidades de cada una de ellas.

Por lo anterior, el objetivo del presente trabajo fue evaluar el efecto de la intensidad de la luz en la productividad de biomasa, lípidos y perfil de ácidos grasos de la microalga Nannochloropsis oculata, en cultivos en lote alimentados en laboratorio para potencializar su uso en la producción de biodiésel.

\section{MATERIALES Y MÉTODOS}

\section{Cultivo}

La cepa $N$. oculata se obtuvo del Departamento de Acuicultura del Centro de Investigación Científica y de Educación Superior de Ensenada, B.C. (CICESE). Los cultivos se llevaron a cabo por triplicado en sistemas por lote alimentado, en matraces Erlenmeyer de $1 \mathrm{~L}$ por un periodo de $21 \mathrm{~d}$. Se mantuvieron bajo condiciones controladas de temperatura a $25^{\circ} \mathrm{C}, \mathrm{pH}$ de 8 , ajustado a través de la adición de gas carbónico $\left(\mathrm{CO}_{2}\right)$ y medido con potenciómetro electrónico Waterproof $\mathrm{pH}$ tester 30 . Se utilizó luz artificial continua con intensidades de 80, 200, 400 y $650 \mu \mathrm{mol} \mathrm{E} / \mathrm{m}^{2} / \mathrm{s}$ proporcionadas con lámparas de luz fría. Los cultivos fueron mantenidos con medio f/2 de Guillard y Ryther (1962) con una composición de $75 \mathrm{mg} / \mathrm{L} \mathrm{KNO}_{3}$, $5.65 \mathrm{mg} / \mathrm{L} \mathrm{NaH}_{2} \mathrm{PO}_{4} .2 \mathrm{H}_{2} \mathrm{O}, 4.360 \mathrm{mg} / \mathrm{L}$ EDTA. $\mathrm{Na}_{2}$, $3.150 \mathrm{mg} / \mathrm{L} \mathrm{FeCl}_{3} .6 \mathrm{H}_{2} \mathrm{O}, 0.010 \mathrm{mg} / \mathrm{L} \mathrm{CuSO}_{4} .5 \mathrm{H}_{2} \mathrm{O}$, $0.022 \mathrm{mg} / \mathrm{L} \mathrm{ZnSO}_{4} .7 \mathrm{H}_{2} \mathrm{O}, 0.010 \mathrm{mg} / \mathrm{L} \mathrm{CoCl}_{2} .6 \mathrm{H}_{2} \mathrm{O}$, $0.180 \mathrm{mg} / \mathrm{L} \quad \mathrm{MnCl}_{2} .4 \mathrm{H}_{2} \mathrm{O}, 0.006 \mathrm{mg} / \mathrm{L}$ $\mathrm{Na}_{2} \mathrm{MoO}_{4} \cdot 2 \mathrm{H}_{2} \mathrm{O}, 2 \mu \mathrm{g} / \mathrm{L}$ cianocobalamina cristalina (B12), $0.100 \mathrm{mg} / \mathrm{L}$ tiamina (B1) y $0.001 \mathrm{mg} / \mathrm{L}$ biotina. Los nutrientes se adicionaron cada $72 \mathrm{~h}$.

La densidad celular se determinó con una cámara de Neubauer de $0.1 \mathrm{~mm}$ de profundidad y microscopio marca Leica Galen III. Se tomaron muestras cada $24 \mathrm{~h}$, además se mantuvo un flujo de aire de $5.2 \mathrm{~L} / \mathrm{min}$, con lo que se aseguró la suspensión de la microalga en el medio de cultivo. Al finalizar el cultivo, la biomasa fue separada por centrifugación a $4000 \mathrm{~g}$ por $15 \mathrm{~min}$.

En todos los tratamientos se determinó la velocidad específica de crecimiento (1/d), densidad celular $(\mathrm{cel} / \mathrm{mL})$ y productividad de biomasa $(\mathrm{g} / \mathrm{L} / \mathrm{d})$.

\section{Extracción de lípidos}

Los lípidos fueron extraídos por el método de Folch et al. (1957) modificado. Se pesó $1 \mathrm{~g}$ de bioma- sa triturada y seca, se adicionaron $100 \mathrm{~mL}$ de mezcla de solventes hexano-metanol $80-20 \% \mathrm{v} / \mathrm{v}$, se agitó por $1 \mathrm{~h}$ a temperatura de $45-50{ }^{\circ} \mathrm{C}$. Posteriormente, se dejó enfriar a temperatura ambiente y se separó la biomasa con filtros de $1.5 \mu \mathrm{m}$ de poro. Se adicionó una mezcla de éter de petróleo-agua $50-50 \% \mathrm{v} / \mathrm{v}$ y se separaron los lípidos de interés, recuperando la fase etérea. El disolvente fue evaporado a $50{ }^{\circ} \mathrm{C}$ y el contenido de lípidos se cuantificó gravimétricamente de acuerdo con la ecuación 1.

$\% \mathrm{~L}=\left(\frac{\text { g lípidos recuperados }}{g \text { biomasa } \sec a}\right) \times 100$

En todos los tratamientos se determinó el porcentaje en peso de lípidos ( $\%$ L) y la productividad $(\mathrm{g} / \mathrm{L} / \mathrm{d})$.

\section{Contenido de ácidos grasos}

La determinación de ácidos grasos se basó en el método de Sánchez-Machado et al. (2009). Se pesaron $0.5 \mathrm{~g}$ de biomasa seca y triturada, se adicionaron $2 \mathrm{~mL}$ de tolueno y $3 \mathrm{~mL}$ de $\mathrm{HCl}$ metanólico al $5 \%$ $(\mathrm{v} / \mathrm{v})$. Las muestras fueron agitadas y colocadas en baño maría a $70{ }^{\circ} \mathrm{C}$ por $2 \mathrm{~h}$. Posteriormente, fueron enfriadas a temperatura ambiente y se les adicionó $3 \mathrm{~mL}$ de solución de $\mathrm{K}_{2} \mathrm{CO}_{3}$ al $6 \%$ y $2 \mathrm{~mL}$ de tolueno, se agitaron y se centrifugaron por $5 \mathrm{~min}$ a $2400 \mathrm{rpm}$. Se separó la fase orgánica, misma que se secó con $\mathrm{Na}_{2} \mathrm{SO}_{4}$ anhidro. Un $\mu \mathrm{L}$ de muestra fue inyectado en el cromatógrafo de gases (Varian CP-3800), se utilizó un detector de ionización de flama a una temperatura constante de $260{ }^{\circ} \mathrm{C}$. La cromatografía se realizó en una columna Varian CP 7485 de $25 \mathrm{~m}$ de longitud y $0.25 \mathrm{~mm}$ de diámetro, se utilizó helio como gas de arrastre (velocidad de flujo de $0.7 \mathrm{~mL} / \mathrm{min}$ ). El horno fue programado para variar de $50{ }^{\circ} \mathrm{C}$ a $220{ }^{\circ} \mathrm{C}$ a una velocidad de 4 ${ }^{\circ} \mathrm{C} / \mathrm{min}$. La identificación de los ácidos grasos en forma de metil ésteres se realizó por comparación del tiempo de retención contra el estándar Supelco 37 (Sigma-Aldrich), que fue analizado en las mismas condiciones. El contenido de ácidos grasos se reportó como el porcentaje relativo al total de ácidos grasos identificados en la muestra.

\section{Análisis estadístico}

Se realizó un análisis de varianza y cuando hubo diferencias, se utilizó entonces la prueba de la diferencia honestamente significativa (HSD, por sus siglas en inglés) de Tukey con un nivel significancia de $\mathrm{p}<0.05$. 


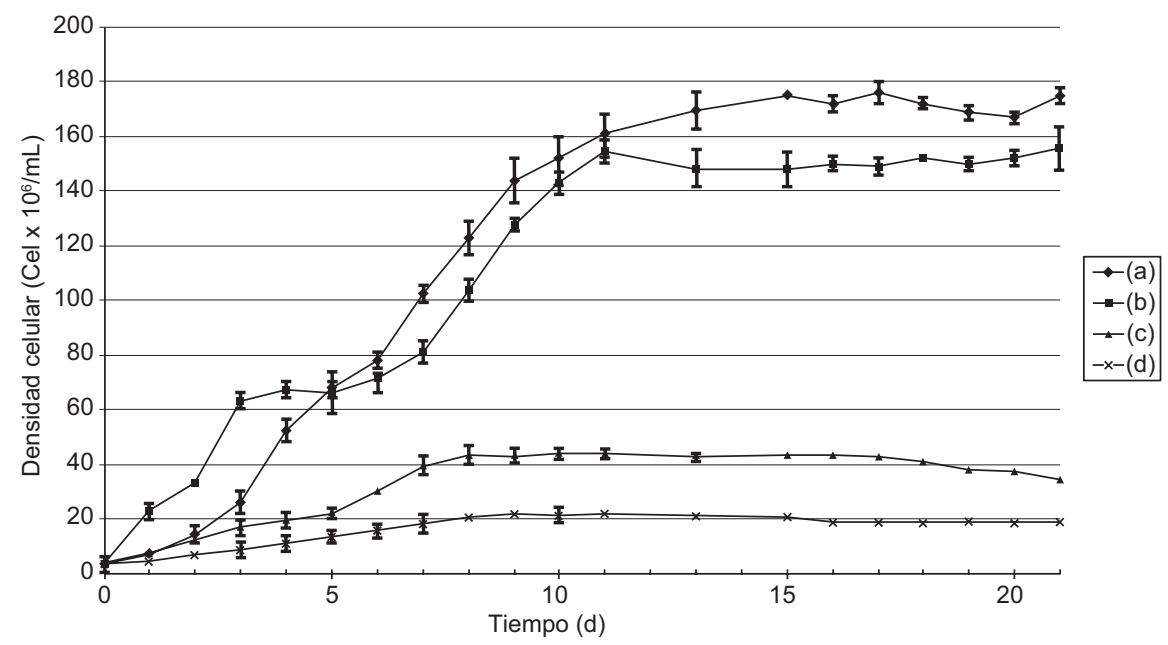

Fig. 1. Cinética de crecimiento del cultivo de Nannochloropsis oculata en cultivos en lote alimentados con intensidades de luz: $650 \mu \mathrm{mol} \mathrm{E} / \mathrm{m}^{2} / \mathrm{s}$ (a), $400 \mu \mathrm{mol} \mathrm{E} / \mathrm{m}^{2} / \mathrm{s}$ (b), 200 $\mu \mathrm{mol} \mathrm{E} / \mathrm{m}^{2} / \mathrm{s}(\mathrm{c}), 80 \mu \mathrm{mol} \mathrm{E} / \mathrm{m}^{2} / \mathrm{s}(\mathrm{d})$. Las barras de error en la gráfica representan la desviación estándar, $\mathrm{n}=9$

\section{RESULTADOS Y DISCUSIÓN}

\section{Cultivo}

La microalga $N$. oculata fue cultivada a irradiancias de $80,200,400$ y $650 \mu \mathrm{mol} \mathrm{E} / \mathrm{m}^{2} / \mathrm{s}$. Durante su desarrollo se determinó la velocidad específica de crecimiento ( $1 / \mathrm{d})$, densidad celular $\left(\mathrm{x} 10^{6} \mathrm{cel} / \mathrm{mL}\right)$ y la productividad de la biomasa $(\mathrm{g} / \mathrm{L} / \mathrm{d})$. Los resultados indicaron que $N$. oculata fue capaz de reproducirse en todas las intensidades de luz, sin presentarse un efecto de fotoinhibición por la irradiancia. En la figura 1 se puede observar que al incrementarse el nivel de irradiancia, aumentó la densidad celular de los cultivos, las más elevadas estuvieron en los tratamientos de 650 y $400 \mu \mathrm{mol} \mathrm{E} / \mathrm{m}^{2} / \mathrm{s}$, con valores de $175 \pm$ 1.83 y $148 \pm 1.97 \times 10^{6} \mathrm{cel} / \mathrm{mL}$, respectivamente. Mientras que las densidades más bajas se registraron en los tratamientos de 80 y $200 \mu \mathrm{mol} \mathrm{E} / \mathrm{m}^{2} / \mathrm{s}$, con valores de $21 \pm 1.12$ y $43 \pm 1.84 \times 10^{6} \mathrm{cel} / \mathrm{mL}$, respectivamente. Un comportamiento similar se observó en las velocidades de crecimiento $(\mu)$. Los valores más altos se obtuvieron en los cultivos mantenidos a 650 y $400 \mu \mathrm{mol} \mathrm{E} / \mathrm{m}^{2} / \mathrm{s}$ con $0.3432 \pm 0.08$ y $0.3299 \pm 0.021 / \mathrm{d}$, respectivamente (Cuadro I). Estos resultados concuerdan con lo encontrado por Huerlimann et al. (2010), quienes a una intensidad de luz de $700 \mu \mathrm{mol} \mathrm{E} / \mathrm{m}^{2} / \mathrm{s}$ registraron en Nannochloropsis sp., una tasa de crecimiento de 0.41 1/d. De acuerdo con estos resultados, el aumento en la intensidad de luz favoreció la densidad celular y la velocidad específica de crecimiento celular. Lo anterior se debió a un incremento en su capacidad de fijar carbono a través de la fotosíntesis (Lobban et al. 1985). Sin embargo, si las intensidades de luz se incrementaran a niveles más elevados, la actividad fotosintética podría sufrir un retardo y/o disminución por inhibición en la producción y foto-oxidación de sus pigmentos (Ouyang et al. 2015).

Por otra parte $N$. oculata logró adaptarse a condiciones de baja intensidad lumínica. Se registró un

CUADRO I. PARÁMETROS CINÉTICOS DE LOS DIFERENTES CULTIVOS

\begin{tabular}{lccc}
\hline $\begin{array}{l}\text { Intensidad de la luz } \\
\left(\mu \mathrm{mol} \mathrm{E} / \mathrm{m}^{2} / \mathrm{s}\right)\end{array}$ & $\begin{array}{c}\text { Velocidad específica } \\
\text { de crecimiento }(1 / \mathrm{d})\end{array}$ & $\begin{array}{c}\text { Densidad celular } \\
\left(\mathrm{x} 10^{6} \mathrm{cel} / \mathrm{mL}\right)\end{array}$ & $\begin{array}{c}\text { Productividad de la } \\
\text { biomasa }(\mathrm{g} / \mathrm{L} / \mathrm{d})\end{array}$ \\
\hline 80 & $0.197 \pm 0.03^{\mathrm{a}}$ & $21 \pm 1.12$ & $0.025 \pm 0.0004^{\mathrm{a}}$ \\
200 & $0.295 \pm 0.01^{\mathrm{a}}$ & $43 \pm 1.84$ & $0.051 \pm 0.0007^{\mathrm{b}}$ \\
400 & $0.329 \pm 0.02^{\mathrm{b}}$ & $148 \pm 1.97$ & $0.073 \pm 0.0007^{\mathrm{c}}$ \\
650 & $0.343 \pm 0.08^{\mathrm{b}}$ & $175 \pm 1.83$ & $0.091 \pm 0.0007^{\mathrm{d}}$ \\
\hline
\end{tabular}

*Letras diferentes en la misma columna representa diferencia significativa $(\mathrm{p}<0.05), \pm$ desviación estándar 
crecimiento positivo debido la habilidad que tiene de modificar su metabolismo. La microalga logró estimular su capacidad de absorción a bajas intensidades de luz a través de un incremento en el contenido de clorofila (Richardson et al. 1983) u otros pigmentos involucrados en la actividad fotosintética. En todos los casos, la tendencia de los parámetros cinéticos incidió directamente en la productividad de la biomasa.

En relación con el contenido de lípidos se puede observar en la figura 2 que es afectado positivamente al incrementar la intensidad de luz. Se alcanzó hasta un $44.44 \pm 0.76 \%$ en peso en la biomasa obtenida en condiciones de irradiancia de $650 \mu \mathrm{mol} \mathrm{E} / \mathrm{m}^{2} / \mathrm{s}$, lo cual es el doble de lo obtenido a $80 \mu \mathrm{mol} \mathrm{E} / \mathrm{m}^{2} / \mathrm{s}$ con un $21.17 \pm 1.08 \%$ de lípidos. Estos resultados son similares a los reportados por diversos autores, van desde un 6.6 hasta un $46.7 \%$ en peso de lípidos acumulados para esta misma especie (Renaud et al. 1991, Chiu et al. 2009, Su et al. 2011). La productividad de los lípidos (Fig. 3) mostró un comportamiento similar, donde, en el cultivo a $650 \mu \mathrm{mol} \mathrm{E} / \mathrm{m}^{2} / \mathrm{s}$ se logró una productividad de $0.040 \pm 0.0005 \mathrm{~g} / \mathrm{L} / \mathrm{d}$, ocho veces mayor a la obtenida en el cultivo de

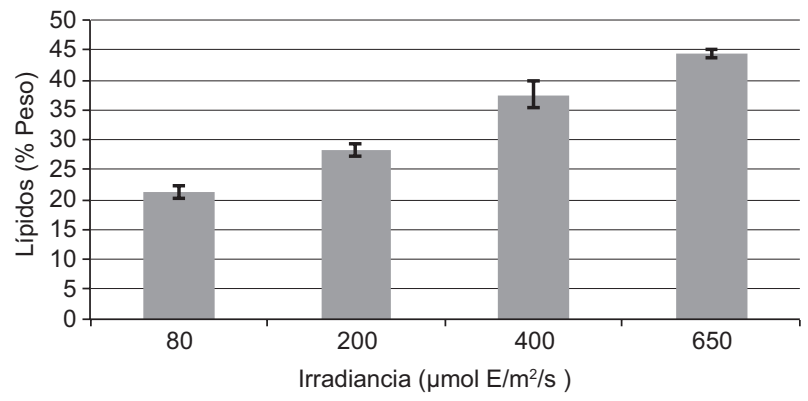

Fig. 2. Contenido de lípidos en la biomasa de Nannochloropsis oculata a diferentes intensidades de luz. Las barras de error en la gráfica representan la desviación estándar, $\mathrm{n}=9$

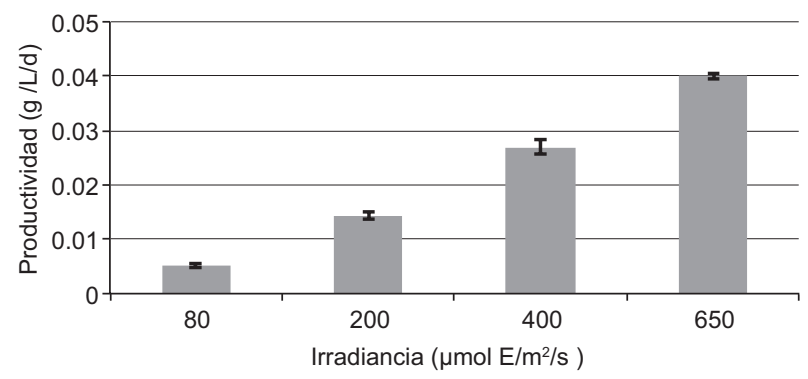

Fig. 3. Productividad de los lípidos en la biomasa de Nannochloropsis oculata a diferentes intensidades de luz. Las barras de error en la gráfica representan la desviación estándar, $\mathrm{n}=9$ menor intensidad de luz de $0.0052 \pm 0.0004 \mathrm{~g} / \mathrm{L} / \mathrm{d}$. Estos resultados concuerdan con los reportados por Rodolfi et al. (2003) y Griffiths et al. (2012), quienes muestran productividades de lípidos similares de 0.061 y $0.063 \mathrm{~g} / \mathrm{L} / \mathrm{d}$, respectivamente. Este incremento puede atribuirse a que: a) la fotosíntesis se ve favorecida por la irradiancia, con lo que se promueve la conversión de los nutrientes en lípidos y b) a que la síntesis de lípidos está coordinada con la síntesis de carotenoides, que son esterificados y almacenados como lípidos en el citoplasma. Estos carotenoides los producen las microalgas a altas intensidades de luz como respuesta a la prevención de daño foto oxidativo (Baker 2006).

Por otra parte, el perfil de ácidos grasos indicó que la microalga tiene como componentes lipídicos a los ácidos grasos saturados mirístico C14:0, pentadecanoico C15:0, palmítico C16:0 y esteárico C18:0 (Cuadro II). Los ácidos grasos saturados e insaturados sintetizados no presentaron variaciones en cuanto a su composición, pero sí se encontró una reducción significativa $(\mathrm{p}<0.05)$ en el contenido de $\operatorname{AEP}(3.34 \pm 0.3)$ a alta intensidad de luz en relación con la registrada a baja intensidad $(5.6 \pm 0.4)$. Esto concuerda con lo mencionado por Volkman et al. (1993), quienes indicaron que las microalgas del género Nannochloropsis, elaboran principalmente ácido palmítico (C16:0), ácido palmitoleico (C16:1n-7) y ácido eicosapentaenoico (C20:5n-3) en condiciones de cultivo similares a $80 \mu \mathrm{mol} \mathrm{E} /$ $\mathrm{m}^{2} / \mathrm{s}$, en medio f/2 de Guillard y Ryther (1962). Lo anterior se puede deber a que el AEP se encuentra principalmente en los componentes estructurales de la membrana de la célula como una especie de lípido polar, cuenta con cinco dobles enlaces, lo que facilita la transferencia de electrones a través de sus enlaces dobles de carbono-carbono y que ocasionan su oxidación con mayor facilidad (Rodolfi et al. 2009). Esto también fue reportado por Arts et al. (2009), quienes indicaron que en Nannochloropsis sp., el grado de insaturación de los ácidos grasos disminuye con el incremento de la intensidad de luz, especialmente el porcentaje total de ácidos grasos n-3, de 29 a $8 \%$ de los ácidos grasos totales, debido principalmente a la reducción del AEP. La reducción o pérdida de dicho ácido ocasiona una mayor estabilidad oxidativa de los lípidos, lo que proporciona un biodiésel de mayor resistencia a la oxidación. Por otro lado, las altas intensidades aumentaron la saturación, particularmente al inducir la acumulación de C16:0, estos resultados concuerdan con los de Tonon et al. (2002) y Pal et al. (2011). 
CUADRO II. PORCENTAJES DE ÁCIDOS GRASOS (AG) RELATIVOS AL TOTAL DE ÁCIDOS GRASOS (TAG) PRESENTES EN Nannochloropsis oculata A DIFERENTES INTENSIDADES DE LUZ

\begin{tabular}{lrrrr}
\hline & \multicolumn{4}{c}{ AG/TAG $(\%$ Peso $)$} \\
\cline { 2 - 5 } Intensidad de luz $\mu \mathrm{mol} \mathrm{E} / \mathrm{m}^{2} / \mathrm{s}$ & \multicolumn{1}{c}{650} & \multicolumn{1}{c}{400} & \multicolumn{1}{c}{200} & \multicolumn{1}{c}{80} \\
\hline Ácido mirístico C14:0 & $6.68 \pm 0.18$ & $7.35 \pm 0.36$ & $7.65 \pm 0.03$ & $8.25 \pm 0.34$ \\
Ácido pentadecanoico C15:0 & $0.61 \pm 0.01$ & $0.63 \pm 0.23$ & $0.58 \pm 0.01$ & $0.52 \pm 0.34$ \\
Ácido palmitíco C16:0 & $38.09 \pm 0.19$ & $38.70 \pm 0.15$ & $38.56 \pm 0.52$ & $39.46 \pm 0.05$ \\
Ácido palmitoleico C16:1n-7 & $30.68 \pm 0.92$ & $30.84 \pm 0.32$ & $29.88 \pm 0.22$ & $29.27 \pm 0.25$ \\
Ácido sapiénico C16:1n-10 & $0.60 \pm 0.03$ & $0.57 \pm 0.02$ & $0.56 \pm 0.18$ & $0.59 \pm 0.01$ \\
Ácido esteárico C18:0 & $0.92 \pm 0.07$ & $0.63 \pm 0.06$ & $0.62 \pm 0.13$ & $0.63 \pm 0.01$ \\
Ácido oleico C18:1 n-9 & $16.38 \pm 0.99$ & $13.12 \pm 0.08$ & $12.19 \pm 0.08$ & $12.54 \pm 0.58$ \\
Ácido linoléico C18:2n-6 & $1.31 \pm 0.11$ & $1.14 \pm 0.06$ & $1.75 \pm 0.24$ & $0.66 \pm 0.05$ \\
Ácido araquidónico C20:4n-3 & $1.33 \pm 0.40$ & $2.96 \pm 0.07$ & $2.75 \pm 0.16$ & $2.48 \pm 0.16$ \\
Ácido eicosapentaenoico C20:5n-3 & $3.34 \pm 0.27$ & $4.06 \pm 0.14$ & $5.46 \pm 0.09$ & $5.60 \pm 0.40$ \\
Ácidos saturados & $46.32 \pm 0.11$ & $47.31 \pm 0.20$ & $47.41 \pm 0.17$ & $48.86 \pm 0.18$ \\
Ácidos insaturados & $53.67 \pm 0.45$ & $52.69 \pm 0.11$ & $52.59 \pm 0.16$ & $51.14 \pm 0.24$ \\
\hline
\end{tabular}

\pm desviación estándar

\section{CONCLUSIONES}

Los resultados indicaron que las productividades de la biomasa y de los lípidos fueron afectadas positivamente con el incremento en la intensidad de luz. También fue favorecida la composición lipídica. Se encontró una reducción en los porcentajes de ácidos grasos poliinsaturados, lo que permite obtener un biodiésel de mayor calidad. La producción y composición de biomasa se mantiene constante a partir del día 13 de cultivo. Al escalarse a nivel industrial, la cosecha se realizaría en este tiempo, por lo que el gasto energético se reduciría, lo que disminuiría el costo del proceso con la máxima obtención de lípidos para producción de biocombustibles.

\section{AGRADECIMIENTOS}

Los autores agradecen al Instituto Tecnológico de Sonora los recursos otorgados para llevar a cabo esta investigación a través del Programa de Fomento y Apoyo a Proyectos de Investigación (PROFAPI).

\section{REFERENCIAS}

Arts M. T., Brett M. T., Kainz M. y Eds J. (2009). Lipids in aquatic ecosystems. Springer Science Business Media. Nueva York, EUA, 367 pp.

Baker N. R. (2006). Photosynthesis and the environment. Kuwer Academic Publishers, Nueva York, EUA, 491 pp.
Chacón C., Andrade C. y Cardenas C. (2004). Uso de Chlorella sp. y Scenedesmus sp. en la remoción de nitrógeno, fósforo y DQO de aguas residuales urbanas de Maracaibo, Venezuela. Bol. Centro Invest. Biol. 38 (2), 94-108.

Chiu S. Y., Kao C. Y., Tsai M. T., Ong S. C., Chen C. H. y Lin C. S. (2009). Lipid accumulation and $\mathrm{CO}_{2}$ utilization of Nannochloropsis oculata in response to $\mathrm{CO}_{2}$ aeration. Bioresource Technol. 100 (2), 833-838. DOI: 10.1016/j.biortech.2008.06.061

Chisti Y. (2007). Biodiesel from microalgae. Biotechnol. Adv. 25 (3), 294-306.

DOI: $10.1016 /$ j.biotechadv.2007.02.001

Folch J., Lees M. y Stanley G.H.S. (1957). A simple method for the insolation and purification of total lipids from animal tissues. J. Biol. Chem. 226 (1), 497-509.

Garibay A., Vázquez-Duhalt R., Sánchez M. Serrano S. y Carreón M. A. (2009). Biodiesel a partir de microalga. Biotecnología 13 (3), 43-48.

Griffiths M. J., Van Hille R. P. y Harrison S. T. (2012). Lipid productivity settling potential and fatty acid profile of 11 microalgal species grown under nitrogen replete and limited conditions. J. Appl. Phycol. 24 (5), 989-1001.

DOI: $10.1007 / \mathrm{s} 10811-011-9723-\mathrm{y}$

Guillard R. R. L. y Ryther J. H. (1962). Studies on marine diatoms: I. Cyclotella nana Hustedt and Denotula confervaceae (Cleve) Gran. Can. J. Microbiol. 8 (2), 229-239. DOI: 229-239, 10.1139/m62-029

Hossain S. y Salleh A. (2008). Biodiesel fuel production from algae as renewable energy. Am. J. Biochem. Biotechnol. 4 (3), 250-254.

DOI: 10.3844/ajbbsp.2008.250.254 
Hu Q., Sommerfeld M., Jarvis E. E., Ghirardi M. L., Posewitz M. C., Seibert M. y Darzins A. (2008). Microalgal triacylglycerols as feedstocks for biofuel production: perspectives and advances. Plant J. 54 (4), 621-639. DOI: 10.1111/j.1365-313X.2008.03492.x

Huerlimann R., De Nys R. y Heimann K. (2010). Growth, lipid content, productivity, and fatty acid composition of tropical microalgae for scale-up production. Biotechnol. Bioeng. 107 (2), 245-257.

DOI: 10.1002/bit.22809

Lobban C. S., Harrison P. J. y Duncan M. J. (1985). The physiological ecology of seaweeds. Cambridge, University Press, Nueva York, EUA, 242 pp.

Mandal S. y Mallick N. (2009). Microalga Scenedesmus obliquus as a potential source for biodiesel production. Appl. Microbiol. Biotechnol. 84(2), 281-291. DOI: 10.1007/s00253-009-1935-6

Ouyang Y., Zhao Y., Sun S., Hu Ch. y Ping L. (2015). Effect of light intensity on the capability of different microalgae species for simultaneous biogas upgrading and biogas slurry nutrient reduction. Int. Biodeter. Biodegr. 104, 157-163. DOI: 10.1016/j.ibiod.2015.05.027

Pal D., Khozin-Goldberg I., Cohen Z. y Boussiba S. (2011). The effect of light, salinity, and nitrogen availability on lipid production by Nannochloropsis sp. Appl. Microbiol. Biotechnol. 90 (4), 1429-1441. DOI: 10.1007/s00253-011-3170-1

Renaud S. M., Parry D. L., Thinh L. V., Kuo C., Padovan A. y Sammy N. (1991). Effect of light intensity on the proximate biochemical and fatty acid composition of Isochrysis sp. and Nannochloropsis oculata for use in tropical aquaculture. J. Appl. Phycol. 3 (1), 43-53. DOI: $10.1007 / \mathrm{BF} 00003918$

Rezanka T., Lukavsky J., Nedbalova L. y Sigler K. (2011). Effect of nitrogen and phosphorus starvation on the polyunsaturated triacylglycerol composition, including positional isomer distribution, in the alga Trachydiscus minutus. Phytochem. 72 (18), 2342-2351.

DOI: 10.1016/j.phytochem.2011.08.017

Richardson K., Beardall J. y Raven J. (1983). Adaptation of unicellular algae to irradiance: an analysis of strategies. New Phytol. 93 (2), 157-191.

DOI: 10.1111/j.1469-8137.1983.tb03422.x

Rodolfi L., Chini Zittelli G., Barsanti L., Rosati G. y Tredici M. (2003). Growth medium recycling in Nannochloropsis sp. mass cultivation. J. Bioeng. 20 (4-20), 243-248. DOI: 10.1016/S1389-0344(03)00063-7

Rodolfi L., Chibi G., Zittelli Ch., Bassi N., Padovani N., Biondi N., Bonini G. y Tredici M. (2009). Microalgae for oil: Strain selection, induction of lipid synthesis and outdoor mass cultivation in a low-cost photobioreactor. Biotechnol. Bioeng. 102 (1), 100-112.

DOI: 10.1002/bit.22033

Sánchez H., Juscamaita J., Vargas J. y Oliveros R. (2008). Producción de la microalga Nannochloropsis oculata (Droop) Hibberd en medios enriquecidos con ensilado biológico de pescado. Bioeng. 7(1-2), 97-100.

Sánchez-Machado D. I., Núñez-Gastélum J. A., ReyesMoreno C., Ramírez-Wong B. y Jaime L. C. (2009). Nutritional quality of edible parts of Moringa oleifera. Food Anal. Methods 3 (3), 175-180.

DOI: $10.1007 / \mathrm{s} 12161-009-9106-\mathrm{Z}$

Simionato D., Sforza E., Carpinelli E. C., Bertucco A., Giacometti G. M. y Morosinotto T. (2011). Acclimation of Nannochloropsis gaditana to different illumination regimes: Effects on lipids accumulation. Biortech. 102 (10), 6026-6032. DOI: 10.1016/j.biortech.2011.02.100

Su C. H., Chien L. J., Gomes J., Lin Y. S., Yu Y. K., Liou J. S. y Syu R. J. (2011). Factors affecting lipid accumulation by Nannochloropsis oculata in a two-stage cultivation process. J. Appl. Phycol. 23 (5), 903-908. DOI: $10.1007 / \mathrm{s} 10811-010-9609-4$

Sukenik A., Beardall J., Kromkamp J. C., Kopecky J., Masojidek J., van Bergeijk S., Gabai S., Shaham E. y Yamshon A. (2009). Photosynthetic performance of outdoor Nannochloropsis mass cultures under a wide range of environmental conditions. Aquat. Microb. Ecol. 56 (2), 297-308. DOI: 10.3354/ame01309

Tonon T., Harvey D., Larso T. R. y Graham I. A. (2002). Long chain polyunsaturated fatty acid production and partitioning to triacylglycerols in four microalgae. Phytochem. 61 (1), 15-24. DOI: 10.1016/S0031-9422(02)00201-7

Volkman J. K., Brown M. R., Dunstan G. A. y Jeffrey S. W. (1993). The biochemical composition of marine microalgae from the class Eustigmatophyceae. J. Phycol. 29 (1), 69-78. DOI: 10.1111/j.1529-8817.1993.tb00281.x

Wijffels R. H. y Barbosa M. J. (2010). An outlook on microalgal biofuels. Science 329 (5993), 796-799.

DOI: 10.1126/science.1189003

Yeesang C. y Cheirsilp B. (2011). Effect of nitrogen, salt, and iron content in the growth medium and light intensity on lipid production by microalgae isolated from freshwater sources in Thailand. Biortech. 102 (3), 3034-3040. DOI: 10.1016/j.biortech.2010.10.013

Yuan X., Kumar A., Sahu A. K. y Ergas S. J. (2011). Impact of ammonia concentration on Spirulina platensis growth in an airlift photobioreactor. Biortech. 102 (3), 3234-3239.

DOI: 10.1016/j.biortech.2010.11.019 